\title{
SPECIES-NEUTRAL DISEASE SURVEILLANCE: A FOUNDATION OF RISK ASSESSMENT AND COMMUNICATION
}

\author{
David R. Franz \\ Midwest Research Institute, MD, USA Kansas State University, KS, \\ USA and the University of Alabama at Birmingham, AL, USA
}

\section{Introduction}

In late June 1999, an unusual number of dead birds were reported in the borough of Queens, New York City. After 6-8 weeks, an unusual number of human cases of encephalitis were first noted in hospitals in the area. The disease was soon diagnosed as St. Louis encephalitis, a mosquito-borne viral encephalitis, the causative agent of which does not kill birds. Approximately 2 weeks after the first human cases, the "St. Louis" outbreak was announced and mosquito control began. Then, 2-3 weeks later, animal disease data and human disease data were integrated, and the true causative agent, West Nile virus, was implicated in both the bird and the human deaths. We will never know if, or exactly how many, lives and dollars might have been saved by knowing 6 weeks earlier that a new, deadly zoonotic arbovirus had been introduced to North America, but experts agree days - and sometimes even hours - make a real difference when dealing with infectious outbreaks. Had we been thinking in terms of disease - wherever it occurs - rather than just human disease, we might have done better.

Two real concerns of the time in which we live are the threat of biological terrorism and the reality of naturally emerging disease. Risk assessment and communication are central to protecting lives and property before and during an outbreak of infectious disease, whether natural or intentional. Becoming aware of any disease outbreak -natural or introduced as early as possible, should be our highest priority. Only when we know what is going on around us can we respond and break the cycle of transmission. Not all microbes have the same impact across species, but the index case may not always be a human. Although Bacillus anthracis is very high on the threat list for terrorist use against humans, it is not typically transmissible and is thus of little concern for livestock. On the 
other hand, a number of arthropod-borne viruses, such as Rift Valley fever virus could have broad implications for both livestock and humans, and even for wildlife. Finally, we face contagious agents, such as avian influenza virus, typically a disease only of birds, which through recombination has become a lethal human pathogen. In addition to West Nile encephalitis and avian flu, other recently emerged viral diseases - severe acute respiratory syndrome (SARS) and monkey pox - occurred first in animals. Whether a disease develops naturally or is introduced by a terrorist, an integrated network of species-neutral surveillance, supported by a deep research base and systems of communication between all sectors, will give days or weeks advanced warning of an outbreak. Therefore, monitoring the space where humans, animals, and microbes meet, while requiring some special tools and strange new agency relationships, will pay huge dividends in lives and resources saved. Disease surveillance and integration of the resulting data must be closely linked to risk assessment and communication activities.

\section{Biological Security and Human Security}

Biological security is fundamental to human security; human security is fundamental to stability in this ever smaller and more connected world. If we are to be as effective as we possibly can be with our risk assessment and communication, we must first understand the playing field. The perceived threats to our biological security cover a broad spectrum world wide; where each of us finds ourselves on that spectrum depends to a great extent in which part of the world we reside. Figure 1 depicts the relative importance of the components of the threat spectrum: the enormous impact of chronic diseases such as HIV/AIDS, malaria, tuberculosis, and hepatitis; the potentially very significant impact of emerging disease, such as SARS and highly-pathogenic influenza; the potentially large impact, but low likelihood of bioterrorism and the emerging concern regarding the exploittation of dual-use biotechnologies to cause harm. The arrows demonstrate regional differences in concern regarding various aspects of the threat spectrum. Therefore, threat perception is related to technological advancement, state of public health and political factors within a given region or country. Where a country or region finds itself on the spectrum will change over time with these factors as well. 


\section{The International Biosecurity Spectrum of Concern}
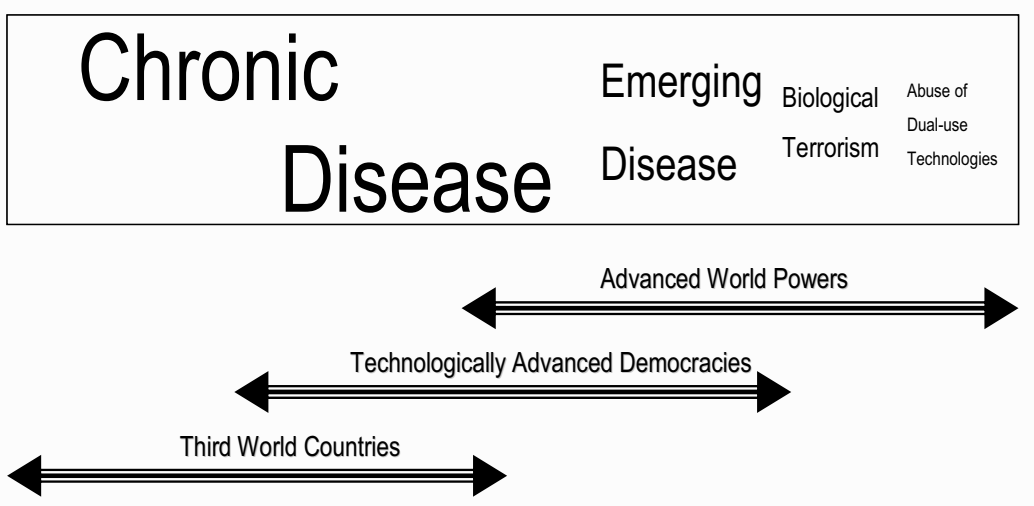

Fig. 1 The spectrum of biosecurity threats. Font within spectrum represents relative health impact. Arrows depict the differing perceptions regarding type of threat in different regions of the world.

\section{Natural vs. Intentional Disease}

Health or economic threats to humans, animals, or both humans and animals caused by biological agents may also be categorized as natural, accidental, or intentional. Variola virus, the causative agent of smallpox, is a threat only to humans; eradicated from the globe nearly 30 years ago, it is no longer a natural threat. It could, however, be reintroduced into the human population intentionally or accidentally. B. anthracis, the causative agent of the disease anthrax, can affect both humans and animals. Because it is not contagious, as is variola, one would not expect there to be an accidental release of significance. There are, however, natural cases throughout the world every year and it is believed to be one of the most important agents for intentional warfare or terrorist release. The foot and mouth disease virus is the most contagious agent known to man. Although it affects only cloven-hoofed animals, it has historically, and could in the future, have an enormous negative economic impact in animal populations, which it infects. It could be introduced naturally, accidentally, or intentionally. Note that, although there are examples, such as variola and the foot and mouth disease virus, which do not cross between humans and animals, the vast majority of agents, which we might have to deal with in the future following natural, accidental, or intentional introduction affect both humans and animals. 


\section{Routes of Exposure}

We often consider aerosol exposure of masses of humans in the context of biological terrorist threats. Granted, most of the classical threat agents anthrax, tularemia, Q fever, etc. must be delivered via aerosol to efficiently infect large numbers of humans. Furthermore, preparing and releasing an agent as an effective aerosol is not a trivial technical accomplishment. Disease may also be introduced via food or drink. Some agents, such as botulinum toxin or the several bacteria, which cause enteric diseases, are well suited to distribution in this way. Certainly there are millions of cases of disease worldwide each year resulting from oral exposure through food or drink. We typically believe, however, that a successful intentional attack on our food supply will be less damaging than a successful aerosol attack on one of our cities. This belief is based on both the route of exposure and the agents typically believed to be ideal for aerosol vs. oral exposure. A third mode of introduction of disease into a population might be through insect or animal vectors. The natural introduction of West Nile encephalitis virus into the United States in 1999 is an example of an agent maintained in nature and transmitted by insects; it could have been introduced by insects or birds. Although there are exceptions, most of the agents of concern from aerosol, food and drink, or vector introduction do not spread easily from person to person or animal to animal; they are not highly contagious. A fourth category of exposure modes is that illustrated by the causative agents of SARS, influenza, smallpox, and foot and mouth disease. These need only be introduced into a population. Because of their propensity to multiply in the respiratory system and/or their tropism for cells of the respiratory tract, they can spread through a population without the aid of man-made aerosols, food, or insects. Therefore, in theory, highly contagious agents need only be introduced to one or a few members of a population to cause infection and even disease in many.

\section{Discovering Disease where it Begins}

In most incidences of widespread disease in a population - whether animal, human, or zoonotic - there is an index, or first, case followed by others. Depending on numerous factors, which we might simply describe as the epidemiological situation, the number of cases will first increase at some rate for a period of time and then, typically, decrease over time as well. Whether the disease is introduced naturally, accidentally, or intentionally, one of the very most important factors is discovering it as early as possible and understanding its spread through the population. Because many diseases of concern to humans are first seen in animals - West Nile 
encephalitis, SARS, monkey pox, H5N1 influenza are recent examples - it is critical that we seek to discover disease in the species of origin. Finding evidence of a zoonotic disease first in animals will very likely allow preventive or prophylactic actions to be taken to protect the human population. The concept of species-neutral disease surveillance acknowledges that we must look for 'disease', not 'human disease' and 'animal disease'. Finally, we live in a much smaller world than we did just a few decades ago. Transportation and travel are such that an outbreak in one part of the world today can impact humans or animals on the other side of the globe tomorrow. Therefore, there is great benefit in discovering an outbreak (1) as early as possible, (2) in the host species of origin, and (3) in the region of origin.

Traditionally, disease surveillance in most countries has involved a Ministry of Health network that monitors human disease and a Ministry of Agriculture, which monitors disease in animals. Even in the 21st century in modern, technologically advanced democracies - it is not uncommon for these two activities to go on in parallel, sometimes discovering the same outbreak in their own species of responsibility, without effective communication between them. During the recent US introductions of West Nile virus and monkey pox, for example, communication between the animal health and human health professionals was less that adequate. Likewise, nations have been reticent to tell other nations that they have discovered a disease outbreak on their soil, fearing negative travel, trade, and economic consequences. The same attitudes and practices have been the norm in many nations and regions throughout the era of modern public health. After the hard-learned lessons described above, there is now evidence of cracks in the walls of parochialism and politics that have slowed our response to disease outbreaks for so many years at great cost in lives and wealth. Knowing that $\sim 75 \%$ of emerging infections diseases and many of those agents traditionally selected for use as weapons are zoonotic - transmissible from animal to man - it only makes sense that we must integrate our disease surveillance efforts. The situational awareness that an integrated disease surveillance program provides must lie at the heart of modern risk assessment and communication, if we are to be prepared to minimize loss of life when disease outbreaks occur.

\section{How Can We Assure Awareness of an Outbreak?}

Both the apparently increasing frequency with which we have faced newly emerging disease in recent years and the attacks experienced in the United States in 2001 have motivated a number of nations to take disease surveillance more seriously. Probably because we believe that we have been 
successful in developing chemical sensors - and we believe that 'bugs' and 'gas' are very similar - and because more engineers have been involved in recent defensive programs than medical and public health experts, environmental monitoring has become and remains popular. The advantage of sensors is that, if they are at the right place and the right time and designed to identify the agent which is used, they could warn us of disease-causing organisms in the air we breathe even before anyone becomes ill. These systems can be placed in the top 40-50 population-dense centers, operating around the clock, all year long, for tens of millions of dollars, or low hundreds. As currently configured, this system will likely not warn us of emerging disease outbreak. A second form of surveillance, data mining, attempts to cast a very wide net to discover, not disease, but human response to a disease outbreak: ambulance calls, over-the-counter medications purchased, emergency room visits, disease-related information sought. Although these passive programs can now be mechanized and might highlight trends and provide us enough information to help us connect the dots, the signal-to-noise problem with such systems complicates their utility. Both the concept of dispersed environmental sensors and passive data mining may be hard to justify for the many years that the threat will exist for the apparently very low-incidence bioterrorism attacks. Amazingly, we believe that the index case of inhalational anthrax was discovered by an astute clinician following the mailing of $B$. anthracislaced letters in fall of 2001. There are a number of clinician-driven surveillance systems undergoing testing today. The greatest challenges of implementing an effective, clinician-driven system are probably (1) the difficulty we find in down-selecting to pick just the right system, (2) failure to have widespread connectivity throughout the various venues in which clinicians see patients, and (3) the time it takes from the clinicians' busy schedule to input data. There are efforts underway to deal with the first problem, by developing one higher-level integrating information system that can take inputs from numerous disparate collection systems. Finally, (4) few clinician-driven disease surveillance systems being tested today integrate human and animal disease data. As with environmental monitoring and data mining, there are trade-offs in implementing clinicianbased surveillance systems. Although we will certainly discover evidence of disease wherever systems are in place, our discovery will come when there is already disease in the population. On the other hand, it may be easier to justify long-term maintenance of clinician-driven surveillance because these systems will be valuable across the entire biothreat spectrum and as preclinical diagnostics improve and find a place in the clinic, or even the home or workplace, we may be able to move ever closer to the 
index case and even the index exposure, the goal of environmental monitoring.

As we think about risk assessment, situational awareness, and communication regarding the biological security threat spectrum, we must address a number of questions. How much information do we really need to prepare or respond effectively? What is the requirement, and value - of these systems for "warning" and for "situational awareness"? How can we down-select from the many systems being developed? How can we make systems truly dual-use? How can we integrate international systems and information? How can we protect patient privacy? What is the appropriate balance of technical and behavioral solutions?

Exactly how to accomplish this important task is not yet clear, but its importance is unquestionable. We must watch that "spot where the animals, humans and bugs collide". Technology would allow it today; we must not let politics or borders stand in the way. Working together, across national boundaries on this, one of the most difficult and important human security issues of our time, will not only protect our citizens from natural disease, but will also build understanding and even trust that will reduce the likelihood that intentional outbreaks will be used against them. 